Vol. 10(7), pp. 130-135, July 2016

DOI: 10.5897/AJPS2016.1405

Article Number: C351F4358995

ISSN 1996-0824

Copyright (C) 2016

African Journal of Plant Science

Author(s) retain the copyright of this article

http://www.academicjournals.org/AJPS

\title{
Evaluation of plant stage dependency of QTLs to homologous and heterologous rust pathogen isolates of barley
}

\author{
Dido A. A. ${ }^{1 \star}$, Freddy Y. K. San ${ }^{2}$ and Rients E. Niks ${ }^{2}$ \\ ${ }^{1}$ Oromia Agricultural Research Institute, Sinana Agricultural Research Center, Plant Biotechnology Research Team, P. \\ O. Box 203,Bale-Robe,South-East Oromia, Ethiopia. \\ ${ }^{2}$ Laboratory of Plant Breeding, Wageningen University, P. O. Box 386, 6700 AJ Wageningen, The Netherlands.
}

Received 15 February, 2016; Accepted 1 May, 2016

\begin{abstract}
A disease test at different leaf layers (plant stages) of homologous rust and heterologous rust species were studied. The result from homologous rust species showed that those quantitative trait loci (QTLs) (Rphq2 and Rphq11) which were effective at seedling stage were also effective across all plant stages with gradually decreasing effect as plants grew older. Rphq3 which had consistent effect in all leaf layers confirmed the same result, that it is a plant stage independent QTL. For heterologous rusts, the effect of Rnhq-V was studied on three rust species; P. hordei-murini (Phm), P. hordei-secalini (Phs) and P. triticina isolate 'Flamingo' at three stages (leaf layers). Infection frequencies are higher at seedling stage and dramatically decrease as plants grow older in all three rust species tested on both SusPtrit and Su-Rnhq-V. The difference between lines tends to be reduced with higher leaf layer in all three tested inappropriate rust species. However, this would be not because of less effectiveness of the Rnhq.
\end{abstract}

Key words: Plant stage, homologous rust, heterologous rust.

\section{INTRODUCTION}

Developmental conditions of the host plant may determine the outcome of pathogen infection; on the other hand, pathogen infection can change the developmental program of the host (Haffner et al., 2015; Grant and Jones, 2009). The influence of plant development on disease resistance is very crucial in understanding of plant-pathogen relationship. Resistance to infectious pathogens appears at different stages of host development, varies with plant age or tissue maturity, may be specific or broad-spectrum and is driven by diverse mechanisms, depending on plant pathogen interactions (DeveleyRiviere and Galiana, 2007). These responses of plants to infectious pathogens include basal response through transcription of genes in response to pathogen-associated molecular pattern recognition, hypersensitive response at the site of infection, systemic acquired resistance making the entire plant resistant to infection, jasmonic acid response and non-host immunity (Boyajyan et al., 2014).

${ }^{*}$ Corresponding author. E-mail: alloaman2010@gmail.com.

Author(s) agree that this article remains permanently open access under the terms of the Creative Commons Attribution License 4.0 International License 
Plants are generally more susceptible to disease in early stage than in late period and this could be due to the fact that there is an increase in resistance through time, with plants already resistance to a pathogen increasing their ability to overcome infection and colonization at a later growth stage (Develey-Riviere and Galiana, 2007). Many studies have been published on this phenomenon and reported for large number of crop plants.

So far different barley (Hordeum vulgaris L.) genotypes to Puccinia hordei were assessed in greenhouse tests at seedling growth stages and in the field at adult plant growth stages (Marcel et al., 2008; Yeo, 2008). For this barley leaf rust $P$. hordei isolate 1.2.1 was used to evaluate the level of partial resistance. Also, a number of quantitative trait loci (QTLs) were found to confer resistance to Puccinia triticina (Pt), Puccinia hordei-murini (Phm), and Puccinia hordei-secalini(Phs) when inoculated on three barley populations (L94xVada, Steptoe x Morex, and Oregon Wolfe Barley populations) (Bettgenhaeuser et al., 2014). From these, diversity of QTLs govern resistance in host system and also indicated that qualitative $\mathrm{R}$ genes are not involved in near nonhost resistance, however, genes conferring partial resistance to adapted pathogens may play role in nonhost resistance (Jafary et al., 2008; Niks, 2014).The effects of QTLs developed from those three barley populations, namely: Rphq2, Rphq11 and Rphq16 are plant stage dependent (Marcel et al., 2007), that they are effective only at seedling stage. Rphq3 on the other hand has a strong and consistent effect at both seedling and adult plant stages. Rnhq is a QTL for nonhost resistance, and was effective to Phm and Phs at seedling stage (Jafary et al., 2006).

Near-isogenic lines (NILs) differing with regard to disease resistance QTLs provide valuable material for a more detailed study into the genetic and molecular dissection of the mechanisms underlies the emergence of disease resistance during host development. Such NILs allows the evaluation of QTL in a nearly uniform genetic background, overcoming the difficulties of identifying QTL phenotypes (Marcel et al., 2007). QTL-NILs do not only provide a better estimate for the effect of single QTL alleles, but also provide a better insight into QTL $\mathrm{x}$ pathogen and QTL x environment interactions.

Therefore, this study used the NILs developed for partial resistance QTLs Rphq2, Rphq3, Rphq11, and Rphq16, and a nonhost resistance QTL, Rnhq with the objective to evaluate whether these QTLs show plant growth stage dependency on resistance to homologous and heterologous rust pathogen isolates.

\section{MATERIALS AND METHODS}

\section{Description of QTL parental lines}

The QTLs that were used in this study were mapped in different
Table 1. Quantitative trait loci near isogenic lines (QTL-NILs) used in this study.

\begin{tabular}{lc}
\hline QTL-NILs & Donor line \\
\hline Rphq2-BC $-B C_{1} S_{1}$ & Vada \\
Rphq3-BC $6 S_{1}$ & Vada \\
Rphq11-s. $F_{2} \cdot B C_{5} S_{1}$ & Steptoe \\
Rphq16-BC $6 S_{1}$ & Dom \\
Qnh.L- $F_{2} \cdot B C_{5} S_{1}$ & L94 \\
Qnh.V.F. $F_{2} \cdot B C_{5} S_{1}$ & Vada \\
\hline
\end{tabular}

barley mapping populations namely L94xVada recombinant inbred lines (RIL) mapping population (Neervoort and Parlevliet, 1978), Steptoe $x$ Morex mapping population (Rasmusson and Wilcoxson,1979) and the Oregon Wolfe Barley (OWB) population (Costa et al., 2001).

\section{Development of a research line and NILs}

Earlier screens of barley accessions for susceptibility to $P$. triticina and $P$. hordei-murini (Phm) allowed identification of several accessions that showed some degree of susceptibility to these rust fungi. Crosses were made between these barley accessions which exhibited relatively high number of pustules and/or high infection types when infected with $P$. triticina and $P h m$. The $F_{2}$ lines for susceptibility to $P$. triticina and $P h m$ at the seedling stage grown to adult plant stage and crossed between the two crossing combinations to obtain double cross (DC) plants. Each DC plant was grown to develop DC-S ${ }_{1}$ lines by selfing. The most susceptible plants within the most susceptible DC-S ${ }_{1}$ lines were selected and selfed for several cycles without selection. Later, susceptible DC-S 5 lines were challenged with $P$. triticina and $P h m$. The DC-S line with the highest number of pustules per leaf and the highest infection type (IT) was selected and named SusPtrit (Sus = Susceptible, P = Puccinia, trit $=$ triticina). SusPtrit was used as a recurrent parent in near isogenic lines (NILs) development program for the QTLs of our interest. (that is, PR QTLs - Rphq2, Rphq3, Rphq11 and Rphq16 and Nonhost resistance QTL- Rnhq).

As stated above, near isogenic lines (NILs) with SusPtrit genetic background (Table 1) and having resistance QTLs, Rphq2, Rphq3, Rphq11, Rphq16 and Rnhq (Rnhq-V and Rnhq-L), were used for this study.

The parental lines for each respective NILs were used as a reference. For Rphq2-BC $C_{5} S_{1}$ and $R p h q 3-B C_{6} S_{1}$ besides SusPtrit and Vada were used as reference, L94, L94-NILs (L94-Rphq2 andRphq3) and Vada-NILs (Vada-rphq2, and -rphq3) were included as well. The QTLs-NILs seeds were sown together with their respective reference lines. The sowing was done two times a week to ensure sufficient plants of each QTLs-NIL and reference lines at the required stage and was extended for eight weeks to have different plant stages. For each QTL-NIL and reference lines, 2 and 3 seeds, respectively were sown in a pot of $14 \mathrm{~cm}$ diameter. At the $8^{\text {th }}$ week, the seeds were sown in boxes $(39 \mathrm{~cm} \times 37 \mathrm{~cm})$. The plants were raised in the greenhouse compartments in three replications.

\section{Inoculum}

Eight different stages of plants for each partial resistance QTL-NIL were inoculated with barley leaf rust $P$. hordei, isolate 1.2.1. For SuRnhq, only three different growth stages (first, second and third leaf 


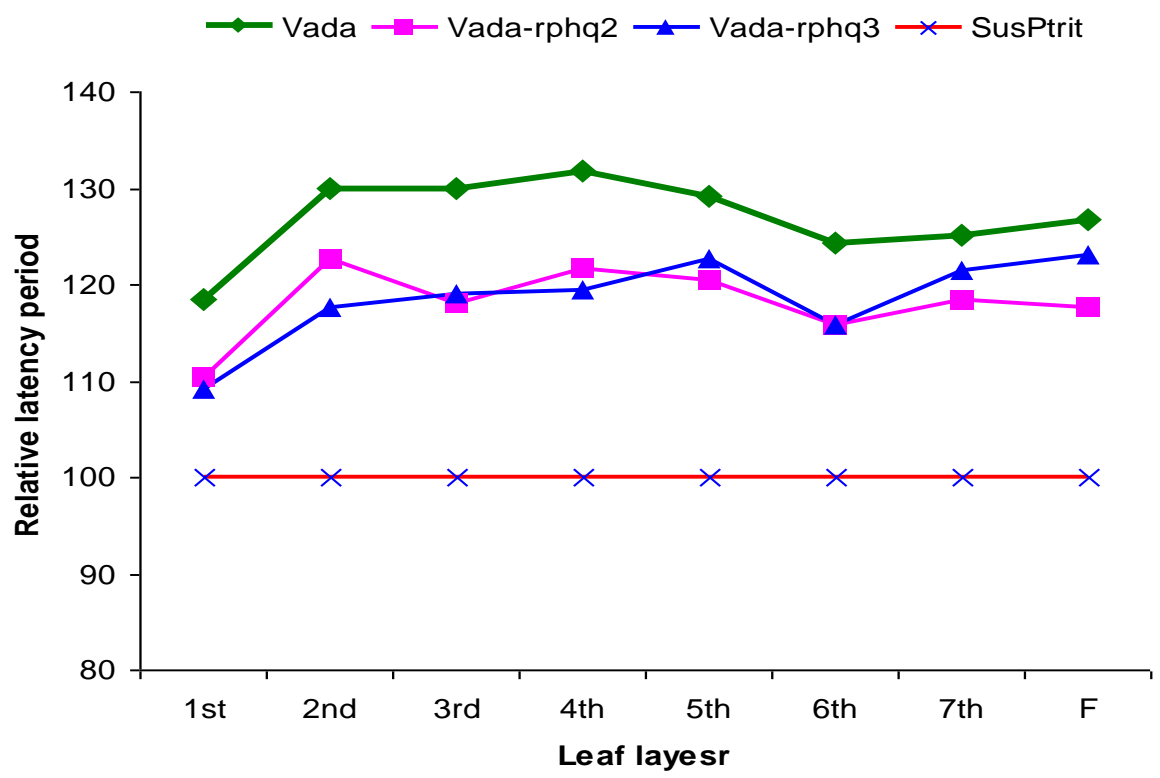

Figure 1. RLP50 of Vada and Vada-NILs relative to SusPtrit infected with $P$. hordei.

stages) were inoculated with $P$. triticina $(P t), P$. hordei-murini $(P h m)$ and $P$. hordei-secalini (Phs) because the adult plants are resistant to these inappropriate rusts.

For inoculation of those plants at each stage, $1 \mathrm{mg}(P$. hordei 1.2.1) and $2 \mathrm{mg}$ (heterologous rusts) of spores diluted 10 times with lycopodium spores were used as inoculum for each pot. Then, the inoculum was sprayed over the plants as uniformly as possible. The plants were then placed in a humidity chamber overnight $(8 \mathrm{~h})$ at $100 \%$ relative humidity in the dark at $18^{\circ} \mathrm{C}$ to allow the spores to germinate. After incubation, the plants were transferred to a greenhouse compartment where the temperature was set at $14 \pm$ $3^{\circ} \mathrm{C}$ with 30 to $70 \%$ relative humidity.

\section{Data collection and analysis}

Five to eight days after inoculation, when the infection flecks appear, observation zones containing proper density of flecks were delimited by marker. The observations started when the susceptible line showed the first mature pustules. The latency period (LP50) of three to five plants per QTL-NILs and two to three plants per parental line per stage in three replications and averages were considered to reflect the level of partial resistance for each QTLNILs and donor lines. For all lines two leaves per pot per plant stages were scored.

For heterologous rusts, the frequency of visible infection sites (VIS; the number of both flecks and pustules per $\mathrm{cm}^{2}$ ) and infection frequency (IF; the number of pustules per $\mathrm{cm}^{2}$ ) following Jafary et al. (2006) were evaluated. Also, the latency period (LP) of the fungi on each plant was calculated. The data collected were analyzed using GenStat 12 th edition statistical software.

\section{RESULTS}

\section{Plant stage dependency of partial resistant QTLs}

All lines tested show an increase in LP from the primary leaf up to the flag leaf. However, the LP of susceptible check, SusPtrit, was lower than the QTL-NILs and other parental lines which carry resistance genes.

LPs for SusPtrit were the shortest, averaging from 192 to $200 \mathrm{~h}$ from first leaf to forth leaf. Compared with SusPtrit, LP differences were slightly larger for QTL-NILs (201 to $240 \mathrm{~h}$ ), Vada NILs (218 to $258 \mathrm{~h}$ ) and L94-NILs (205 to $248 \mathrm{~h}$ ) across all leaf layers (growth stages).

\section{Rphq2}

From first leaf to third leaf layers, Rphq2 has longer RLP than Rphq3 and up to second leaf layers compared to Rphq11 on NILs with SusPtrit genetic background. Its effect starts to gradually decrease from second and fourth leaf layer onwards on L94-Rphq2 and Sus-Rphq2, respectively. In general, the effect of Rphq2 in SusRphq2 and L94-Rphq2 is not significant above forth leaf layers (Figures 1 to 3 ). On the other hand, in Vada background NIL with rphq3, longer LP was observed after six leaf layer due to the presence of Rphq2. Its effect was lower than Rphq3 on first, second and forth leaf layers.

\section{Rphq3}

The effect of this QTL is gradually increased after third leaf stage showing consistent effect in all developmental stages (Figures 1 to 3), except in Vada-rphq2. The effect of Rphq3 was lower than Rphq2 on L94-Rphq2 from first to second leaf layers (Figure 2). It is also, lower than Rphq2 and Rphq11 from first leaf to third leaf layers on Su-Rphq2 and Su-Rphq11, respectively (Figure 3). On the sixth leaf layer, its effect becomes equal to Rphq2 


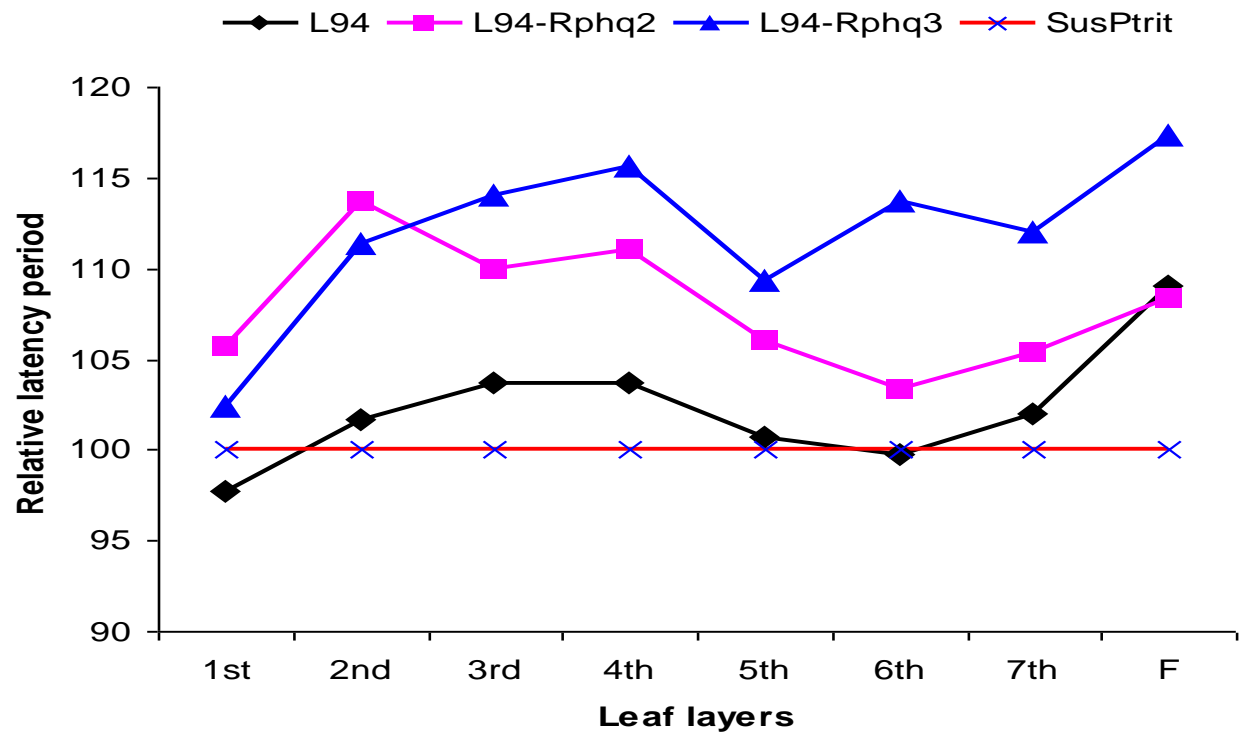

Figure 2. RLP50 of L94 and L94-NILs relative to SusPtrit infected with P. hordei.



Figure 3. RLP50 of Su-Rphq2, Su-Rphq3 and Su-Rphq11 relative to SusPtrit infected with $P$. hordei.

and Rphq11 on Su-Rphq2, and Su-Rphq11, respectively. However, its effect increases afterwards (Figure 3). In Vada-NILs with rphq2, the effect of Rphq3 was higher than that with rphq3 up to forth leaf layers though it has lower effect at third and sixth leaf layer afterwards.

\section{Rphq11}

The effect of Rphq11 on Su-Rphq11 was higher than SuRphq3 from first leaf to third leaf layers (Figure 3). As plants grow higher, the RLP of Su-Rphq11 increased as in other QTLs-NILs.

\section{Plant stage dependency of nonhost resistance QTLs (Rnhq-V)}

Effects of Su-Rnhq-V to the three inappropriate rust fungi were assessed by determining the relative latency period (RLP) and number of macroscopically visible infection sites. The level of infection established by inappropriate rusts rage from immune (no pustules and less than three flecks per $\mathrm{cm}^{2}$ ) to susceptible. Infection frequencies are 
Table 2. RIF of Su-Rnhq-V, SusPtrit, and L94 infected with Pt, Phm and Phs.

\begin{tabular}{lccccccccc}
\hline \multirow{2}{*}{ Lines } & \multicolumn{4}{c}{$\boldsymbol{P}$. Triticina (Flamingo) } & \multicolumn{3}{c}{$\boldsymbol{P}$. hordei-murini } & \multicolumn{3}{c}{$\boldsymbol{P}$. hordei-secalini } \\
\cline { 2 - 10 } & $\mathbf{1}$ & $\mathbf{2}$ & $\mathbf{3}$ & $\mathbf{1}$ & $\mathbf{2}$ & $\mathbf{3}$ & $\mathbf{1}$ & $\mathbf{2}$ & $\mathbf{3}$ \\
\hline Su-Rnhq-V & 66.3 & 38.0 & 33.0 & 37.5 & 22.0 & 18.0 & 39.7 & 33.7 & 25.0 \\
Vada & 2.1 & - & - & - & - & - & 6.0 & 2.0 & - \\
L-94 & 73.1 & 47.0 & 39.5 & 63.0 & 57.0 & 53.0 & 77.3 & 69.0 & 63.0 \\
SusPtrit & 100. & 100.0 & 100.0 & 100.0 & 100.0 & 100.0 & 100.0 & 100.0 & 100.0 \\
\hline
\end{tabular}

Table 3. RLP50 of Su-Rnhq-V, SusPtrit, and L94 infected with Pt, Phm and Phs.

\begin{tabular}{lccccccccc}
\hline \multirow{2}{*}{ Lines } & \multicolumn{1}{c}{$\boldsymbol{P}$. Triticina (Flamingo) } & \multicolumn{1}{c}{$\boldsymbol{P}$. hordei-murini } & \multicolumn{3}{c}{$\boldsymbol{P}$. hordei-secalini } \\
\cline { 2 - 10 } & 1 & 2 & 3 & 1 & 2 & 3 & 1 & 2 & 3 \\
\hline Su-Rnhq-V & 101 & 105 & 103 & 102 & 104 & 103 & 104 & 103 & 103 \\
Vada & - & - & - & - & - & - & 117 & 122 & - \\
L-94 & 103 & 105 & 108 & 103 & 105 & 109 & 101 & 103 & 107 \\
SusPtrit & 100 & 100 & 100 & 100 & 100 & 100 & 100 & 100 & 100 \\
\hline
\end{tabular}

higher at seedling stage and dramatically decrease as plants grow higher in all three rust species tested (Table 2). The uredia size of infected leaves was small and had chlorosis on Su-Rnhq-V NILs at seedling stage as compared to that of SusPtrit.

As shown in Table 2, for infection with $P$. triticina, effect of $R n h q-V$ showed significant effect on Su-Rnhq-V only at second leaf stage. In case of $\mathrm{Phm}$ at first leaf stage, the effect is not significant; however there was positive effect at second leaf stage. It had positive effect on RLP at first and second leaf stage for Phs. However, the effect seems to decrease at second and third leaf stages. In general, the effect of Rnhq-V on Su-Rnhq-V on RLP tends to decrease with increment in leaf layer in all three tested inappropriate rust species. Parental line 'Vada' showed no sporulating uredia on both $P$. triticina and Phm except very few on Phs, as a result RLP was not scored for this line on those two rust species (Table 3).

\section{DISCUSSION}

Adequate standardization of plant age, inoculum density and quality, and environmental conditions are required to recognize true differences in susceptibility. In this research, the environmental conditions during plant growth prior to inoculation, during exposure of inoculated plants to dew, and during post-dew development were sufficiently defined and controlled to provide an acceptable level of variation in disease development attributable solely to environmental factors.

Near-isogenic lines (NILs) differing with regard to disease resistance QTLs provide valuable material for a more detailed study into the genetic and molecular dissection of the mechanisms underlying the emergence of disease resistance during host development. Such NILs allows the evaluation of QTL in a nearly uniform genetic background, overcoming the difficulties of identifying QTL phenotypes (Marcel et al., 2007). QTLNILs do not only provide a better estimate for the effect of single QTL alleles, but also provide a better insight into QTL x pathogen and QTL x environment interactions.

In this study, three most effective QTL-NILs with SusPtrit background, Rphq2, Rphq3, and Rphq11 contributed in resistance to homologous rust were used to evaluate the effect of each QTL at different plant development stages.

As shown in Figure 3, Rphq2 on Su-Rphq2 had higher effect from first to third leaf layers as compared to Rphq3 on Su-Rphq3. Its effect starts to relatively decrease after fourth leaf layers in SusPtrit background NILs. Rphq2 is effective at seedling stage and gradually lose its effect as plant grows higher. The effect of Rphq3 on Su-Rphq3 consistently increases from first leaf layer to adult plant stage. This consistency in effect indicates that this QTL is stage independent. On the other hand, Rphq11 on SuRphq11 had no as such statistically significant difference from Rphq2 and Rphq3 on Su-Rphq2 and Su-Rphq3, respectively. It tended to have an effect intermediate between Rphq2 and Rphq3 (Figure 3).

In previous studies (Niks et al., 2000; Marcel et al., 2008) it was reported that Rphq2 had a strong effect in the seedling stage but almost no effect in adult plant stage, while Rphq3 was effective in seedling and adult plant stages indicating that Rphq3 is plant stage independent. Also it was reported (Yeo, 2008) that, Rphq11 was effective in seedling stage. However, in present study, it was observed that those QTLs which were effective at seedling stage were also effective across all plant stages with gradually decreasing effects 
as plants grew older. Previously, these QTLs were reported to be plant stage dependent because they were mapped at seedlings stage but not at adult stage. Here the QTL-NILs used for this study were only those in which QTLs of interest is in the plant material. So, that the QTLs which were reported to be plant stage dependent may not be as reported due to the fact that QTLs do function throughout the plant stage but its effect is smaller than other QTLs detected in a mapping population. However, the effect of Rphq3 is consistent in all leaf layers observed, indicating that this QTL is plant stage independent as reported in previous studies. As far as Rnhq is concerned, Su-Rnhq-V had positive effect in resistance at first leaf layer; however, its effect seems to decrease as plants grew older.

Generally, as reviewed in detail in Develey-Rivière and Galiana (2007), resistance acquisition during development has been reported for a large number of crops from both monocots and dicots (wheat, rice, maize, soybean, common bean, tomato, grapevine, tobacco). This resistance to diseases associated with major transitions happening during plant life cycle (Kus et al., 2002; Poethig, 2003; Rusterucci et al., 2005; Baurle and Dean, 2006), function of the maturity of tissue or organ (Zeier, 2005), acquired resistance with development (Panter et al., 2002; Rusterucci et al., 2005), the functional regulation of plant resistance $(R)$ genes (Panter et al., 2002; McDowell et al., 2005), induction of defense mechanisms (Cameron and Zaton, 2004; Dong, 2004; Hugot et al., 2004; Xu et al., 2006). In this study, we observed the same phenomenon of resistance at different developmental stage of nearly isogenic barley lines.

\section{Conflict of Interests}

The authors have not declared any conflict of interests.

\section{ACKNOWLEDGMENTS}

This work is part of M.Sc. thesis of the senior author. The study was financially supported by Netherlands Fellowship Program (NFP-AP).

\section{REFERENCES}

Baurle I, Dean C (2006). The timing of developmental transitions in plants. Cell 125:655-664.

Bettgenhaeuser J, Gilbert B, Ayliffe M, Moscou MJ (2014). Nonhost resistance to rust pathogens-a continuation of continua. Front. Plant Sci. 5(664):10-3389

Boyajyan A, Devejyan H, Haykazyan V, Avetisyan G, Khanoyan D (2014). Molecular mechanisms and mediators of the immune response in plants. J. Plant Sci. 2(1):23-30.

Cameron RK, Zaton K (2004). Intercellular salicylic acid accumulation is important for age-related resistance in Arabidopsis to Pseudomonas syringae. Physiol. Mol. Plant Pathol. 65:197-209.

Costa JM, Corey A, Hayes PM, Jobet C, Kleinhofs A, Kopisch-Obusch A, Kramer SF, Kudrna D, Li M, Riera-Lizarazu O, Sato K, Szucs P, Toojinda T, Vales MI, Wolfe RI (2001). Molecular mapping of the
Oregon Wolfe Barleys: a phenotypically polymorphic doubled-haploid population. Theor. Appl. Genet. 103:415-424

Develey-Rivière M, Galiana E (2007). Resistance to pathogens and host developmental stage: a multifaceted relationship within the plant kingdom. New Phytol. 175:405-416.

Dong X (2004). NPR1, all things considered. Curr. Opin. Plant Biol. 7:547-552.

Grant MR, Jones JDG (2009). Hormone (dis)harmony moulds plant health and disease. Science 324:750-752.

Haffner E, Konietzki S, Diederichsen E (2015). Keeping Control: The Role of Senescence and Development in Plant Pathogenesis and Defense. Plants 4:449-488.

Hugot K, Riviere MP, Moreilhon C, Dayem MA, Cozzitorto J, Arbiol G, Barbry P, Weiss C, Galiana E (2004). Coordinated regulation of genes for secretion in tobacco at late developmental stages: association with resistance against oomycetes. Plant Physiol. 134:858-870.

Jafary H, Albertazzi G, Marcel TC, Niks RE (2008). High diversity of genes for nonhost resistance of barley to heterologous rust fungi. Genetics 178, 2327-2339. doi:10.1534/genetics.107.077552

Jafary H, Szabo LJ, Niks RE (2006). Innate nonhost immunity in barley to different heterologous rust fungi is controlled by sets of resistance genes with different and overlapping specificities. Mol. Plant Microbe Interact. 19(11):1270-1279.

Kus JV, Zaton K, Sarkar R, Cameron RK (2002). Age-related resistance in Arabidopsis is a developmentally regulated defense response to Pseudomonas syringae. Plant Cell 14:479-490.

Marcel TC, Varshney RK, Barbieri M, Jafary H, de Kock MJD, Graner A, Niks RE (2007). A high-density consensus map of barley to compare the distribution of QTLs for partial resistance to Puccinia hordei and of defence gene homologues. Theor Appl. Genet 114:487-500.

Marcel TC, Gorguet B, Ta MT, Vels A, Niks RE (2008). The verification of QTLs for partial resistance to Puccinia hordei in NILs of barley confirms an isolate-specific effect. New Phytol. 177:743-755

McDowell JM, Williams SG, Funderburg NT, Eulgem T, Dangl JL (2005). Genetic analysis of developmentally regulated resistance to downy mildew (Hyaloperonospora parasitica) in Arabidopsis thaliana. Mol. Plant Microbe Interact. 18:1226-1234.

Neervoort WJ, Parlevliet JE (1978). Partial resistance of barley to leaf rust, Puccinia hordei. V. Analysis of the components of partial resistance in eight barley cultivars. Euphytica 27:33-39.

Niks RE (2014). How specific is non-hypersensitive host and nonhost resistance of barley to rust and mildew fungi? J. Integr. Agric. 13:244254

Niks RE, Fernández E, Van Haperen B, Bekele AB, Martínez F (2000). Specificity of QTLs for partial and non-host resistance of barley to leaf rust fungi. Acta Phytopathol. Entomol. Hung. 35(1/4):13-21.

Panter SN, Hammond-Kosack KE, Harrison K, Jones JD, Jones DA (2002). Developmental control of promoter activity is not responsible for mature onset of Cf-9B-mediated resistance to leaf mold in tomato. Mol. Plant Microbe Interact. 15:1099-1107.

Poethig RS (2003). Phase change and the regulation of developmental timing in plants. Science 301:334-336.

Rasmusson DC, Wilcoxson RD (1979). Registration of 'Morex' barley. Crop Sci. 19:293

Rusterucci C, Zhao Z, Haines K, Mellersh D, Neumann M, Cameron RK (2005). Age-related resistance to Pseudomonas syringae pv. tomato is associated with the transition to flowering in Arabidopsis and is effective against Peronospora parasitica. Physiol. Mol. Plant Pathol. 66:222-231.

Xu WH, Wang YS, Liu GZ, Chen X, Tinjuangjun P, Pi LY, Song WY (2006). The autophosphorylated Ser686, Thr688, and Ser689 residues in the intracellular juxtamembrane domain of XA21 are implicated in stability control of rice receptor-like kinase. Plant $\mathrm{J}$. 45:740-751.

Yeo KSF (2008). Genetic Dissection of QTLs for partial resistance of barley to Puccinia hordei. MSc, Thesis, Wageningen University, Plant Breeding Department, The Netherlands.

Zeier J (2005). Age-dependent variations of local and systemic defence responses in Arabidopsis leaves towards an avirulent strain of Pseudomonas syringae. Physiol. Mol. Plant Pathol. 66(1):30-39. 